\section{Mechanische Klappen stören den Schlaf}

Viele Patienten fühlen sich offenbar von den Geräuschen ihrer mechanischen Herzklappen so stark gestört, dass sie nicht schlafen können. Das ließen 23\% der Teilnehmer einer Umfrage verlauten, die zwischen 2000 und 2011 vorgenommen wurde. Die meisten der insgesamt 245 befragten Patienten (87\% der Männer und 75\% der Frauen) gaben an, dass sie das Schließen ihrer Klappe hören können. Eine von den $\mathrm{Pa}$ tienten angegebene Methode, sich der störenden Geräusche im Schlaf zu entledigen, war das Schlafen auf der rechten Seite, wodurch die Geräusche abnehmen sollen, gefolgt von Bettdecke über den Kopf legen, Musik hören und Entspannungsübungen.

Oterhals K. EuroHeartCare Kongress 2017

\section{Vitamin D für schwache Herzen?}

Forscher haben untersucht, ob eine dosierte Zufuhr von Vitamin D die Sterblichkeit von Patienten mit Herzinsuffizienz senkt. 400 Patienten mit Herzschwäche und einem Vitamin-D3-Spiegel unter $75 \mathrm{nmol} / 1$ (im Schnitt sogar unter $40 \mathrm{nmol} / \mathrm{l}$ ) nahmen an der Untersuchung teil. 201 erhielten ein Placebopräparat, 199 nahmen über drei Jahre hinweg täglich 4000 IU Vitamin D3 zu sich. Statistisch relevante Unterschiede in der Gesamtmortalität zwischen beiden Gruppen waren nicht festzustellen, sie betrug nach drei Jahren 19,6\% in der Vitamin- und 17,9\% in der Placebogruppe. Dabei hatte die Vitamin-Supplementation die Serumspiegel auf etwa $100 \mathrm{nmol} / \mathrm{l}$ gehoben.

Zittermann A et al. Eur Heart J 2017 (online first)

\section{Von Herzen gesund bleiben}

"Sport ist Mord" - mit dieser Aussage dürften sich Couchpotatoes nun zumindest teilweise bestätigt fühlen, denn Wissenschaftler konnten in einer Studie belegen, dass Extremsport dem Herzen ganz und gar nicht gut tut und sogar die Mortalität erhöht. Zu wieviel Bewegung Sie Ihren Patienten und dabei vor allem den Herzkranken je nach Diagnose raten sollten, lesen Sie neben vielen weiteren spannenden Themen in unserem Schwerpunkt „Kardiologie" ab Seite 24.

Claudia Daniels Redakteurin

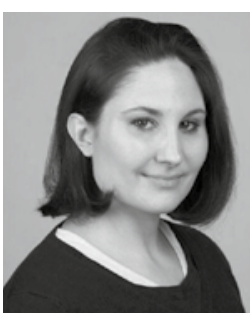

\section{Hypertonie in jungen Jahren erhöht eigenes und familiäres Risiko}

Wenn bei einem Patienten bzw. in dessen Familie Bluthochdruck besteht, scheint es prognostisch sinnvoll zu sein, nach dem Erkrankungsalter zu fragen. Im Vergleich zu einem späten Beginn ist bei einer frühen Manifestation eher zu befürchten, dass der Patient an kardiovaskulären Komplikationen stirbt bzw. dass der Hochdruck vererbt wurde. Darauf deutet eine neue Auswertung der Framingham-Heart-Studie mit insgesamt 1635 Teilnehmern hin. Ausgewertet wurden die Daten von zwei Genera- tionen, bei denen über 60 Jahre hinweg Blutdruckmessungen stattgefunden haben. Wenn ein Elternteil bzw. beide Eltern früh (d.h. vor dem 55. Lebensjahr) an Bluthochdruck erkrankt waren, war das Risiko, selbst Hypertoniker zu werden um den Faktor 2,0 bzw. 3,5 erhöht. Teilnehmer, bei denen mindestens ein Elternteil nach dem 55. Lebensjahr an Bluthochdruck erkrankt war, wiesen kein erhöhtes Risiko auf.

Niiranen TJ et al. BMJ 2017;357:j1949

\title{
Arrhythmiegefahr durch Energy-Drinks?
}

In einer Studie haben Wissenschaftler die Wirkung von Energy-Drinks auf Blutdruck und EKG untersucht. Hierfür hatten sich 18 gesunde Probanden zwischen 18 und 40 Jahren zur Verfügung gestellt, die entweder $946 \mathrm{ml}$ Energy-Drink oder ein anderes koffeinhaltiges Getränk (Kontrollgruppe) gleichen Koffeingehalts zu sich nahmen. Im Anschluss wurde über 24 Stunden mehrfach der Blutdruck gemessen sowie ein EKG durchgeführt. Zwei Stunden nach Aufnahme des Energy-Drinks bzw. des Kontrollgetränks zeigte sich hinsichtlich der Veränderungen der frequenzkorrigier- ten QT-Zeit (QTc) gegenüber dem Ausgangswert ein signifikanter Unterschied zwischen den beiden Gruppen. Der periphere systolische Blutdruck lag in der Versuchs-Gruppe sechs Stunden nach Konsum des Energy-Drinks noch immer 4,72 mmHg über dem Ausgangswert, in der Kontrollgruppe dagegen nur um 0,83 $\mathrm{mmHg}$. Künftig müsse untersucht werden, inwieweit weitere Inhaltsstoffe von EnergyDrinks wie z.B. Taurin für die Veränderungen verantwortlich seien, so die Forscher.

Fletcher EA et al. J Am Heart Assoc. 2017;6:e004448

\section{Leistungssportler haben mehr Plaques}

Eine Untersuchung von 152 extremen Ausdauersportlern ergab, dass bei männlichen Athleten häufiger atherosklerotische $\mathrm{Ab}$ lagerungen in den Koronararterien zu finden sind als bei weniger aktiven Altersgenossen. Fördert exzessiver Sport somit die Entstehung von Arteriosklerose? Das Ergebnis könne man zwar zunächst so auslegen, schreiben die Studienautoren um Ahmed Merghani, London. Doch die bei den Sportlern vorzufindende stabile Struktur der Plaques könnte auf der anderen Seite vor einer PlaqueRuptur und damit vor einem Herzinfarkt schützen. Sportler und Kontrollpersonen waren bzgl. des Alters (zwischen 40 und 82 Jahre), des Geschlechts (70\% Männer) und der Höhe des Framingham-Scores $(3,4 \%)$ vergleichbar. Arteriosklerotische Plaques fanden sich bei den Leistungssportlern häufiger, aber nur bei den männlichen Athleten (44,3 vs. 22,2\%). Ausschließlich bei $11 \%$ der männlichen Sportler wurde in der CT-Angiografie eine moderate bis schwere Koronarverkalkung festgestellt. Auch $\geq 50 \%$ ige Stenosen kamen nur bei den männlichen Athleten vor (7,5\%).

Merghani A et al. Circulation. 2017 (online first) 